\title{
Bemerkungen \\ über die Sehschärfe hochgradig myopischer Augen vor und nach operativer Beseitigung der Linse.
}

\author{
Von \\ Professor Th. Leber \\ in Heidelberg. \\ Mit 4 Figuren im Text.
}

Der vorhergehende Aufsatz hat mir zu einer etwas ausführlicheren Bearbeitung des Gregenstandes Anlass gegeben, welche auch nach den bisher darüber vorliegenden Arbeiten und Bemerkungen von Fukala ${ }^{1}$, Schanz ${ }^{2}$, Schweigger ${ }^{3}$ ), v. Hippel ${ }^{4}$ ), Sattler ${ }^{5}$ ) und Anderen etwas

4) Fukala, Operative Behandlung der höchstgradigen Myopie durch Aphakie. Dieses Archiv XXXVI. 2. S. 234-235. (1890). Ueber die Verbesserung der Sehschärfe bei höchstgradig myopisch gewesenen Aphakischen. Archiv f. Augenheilkunde XXIV. S. 161168. (1892). - Heilung höchstgradiger Kurzsichtigkeit etc. 8. Leipzig u. Wien 1896. S. $54-63$.

2) Fr. Schanz, Ueber die Zunahme der Sehschärfe bei der operativen Beseitigung hochgradiger Kurzsichtigleit. Dieses Arehiv XLI. 1. S. 109-118 (1895).

3) Schweigger, Correction der Myopie durch Aphakie. Sitzungsbericht der ophthalm. Gesellschaft f. 1892. S. 115-118. Ibid. f. 1895. S. 33 (Discussion).

4) v. Hippel, Ueber die operative Behandlung hochgradiger Myopie. Ibid. f. 1895. S. 23-24.

5) Sattler, Ueber die operative Behandlung der hochgradigen Myopie. Ibid. f. 1895, S. 28. 
zur weiteren Aufklärung über die hier in Frage kommenden Verhältnisse beitragen dürfte.

Wenn man von Verbesserung der Sehschärfe nach der Myopie-Operation spricht, so muss man zu unterscheiden suchen, wie weit es sich dabei um eine Verbesserung des Distinctionsvermögens des Auges handelt, welche entweder von einer besseren Functionirung der Netzhaut oder von einer grösseren Schärfe der Netzhautbilder herrühren könnte, oder wie weit man es lediglich mit einer Zunahme der Sehschärfe durch Vergrösserung der Netzhautbilder zu thun hat. Bei der Mehrzahl der Augen ist für einen bestimmten Sehwinkel die Grösse des Netzhautbildes nicht viel verschieden, so dass man sich gewöhnt hat, die Sehschärre schlechthin als Maass für das Distinctionsvermögen des Auges zu betrachten. Dies gilt aber nicht mehr, wenn man das Sehen vor und nach der Myopie-Operation zu vergleichen hat; hier muss Sehschärfe und Distinctionsvermögen auseinander gehalten werden.

Wir bezeichnen bekanntlich als Sehschärfe das Vermögen des Auges, räumliche Dimensionen za unterscheiden, und messen dasselbe durch die Tangente des kleinsten Sehwinkels, unter welchem zwei Punkte der Aussenwelt noch gesondert wahrgenommen werden. Die Sehschärfe ist proportional dem Distinctionsvermögen des Auges und der Grösse der Netzhautbilder oder dem Abstand der Netzhaut vom Knotenpunkt des Auges. Das Distinctionsvermögen ist die Fähigkeit des Auges, zwei Netzhautbilder getrennt zu empfinden; es würde zu messen sein durch den kleinsten Abstand der Centren dieser Bilder, bei welchem sie noch getrennt percipirt werden, kann aber natürlich nur indirect bestimmt werden.

Bei gleichem Distinctionsvermögen ist also die Sehschärfe abhängig von der Grösse der Netzhautbilder, welche zwei Augen, oder ein und dasselbe Auge unter verschiedenen Umständen, von einem unter dem gleichen Sehwinkel 
erscheinenden Gegenstande entwerfen; bei gleich grossen Netzhautbildern giebt die Sehschärfe ein unmittelbares Maass für das Distinctionsvermögen zweier verschiedener Augen ab.

Es ist bekannt, dass Concavgläser verkleinern und Convexgläser vergrössern, weil wir ihr optisches Centrum nicht mit dem des Auges zusammenfallen lassen können, sondern sie in einen gewissen Abstand vom Auge bringen müssen; das Maass dieses Einflusses auf die scheinbare Grösse des G'egenstandes hängt ab von dem Abstande der Gläser vom Auge. Bei den die überwiegende Mehrzahl der Fälle bildenden Refractionsanomalieen geringeren bis höheren Grades ist die verkleinernde oder vergrössernde Wirkung der Correctionsgläser unerheblich und wird daher bei der Bestimmung der Sehschärfe in der Regel ohne Schaden vernachlässigt. Dies ist aber bei sehr hochgradiger Ametropie nicht mehr der Fall; hier muss der Einfluss des angewendeten Glases auf die scheinbare Grösse des Gegenstandes berïcksichtigt werden, und es ist dazu nöthig, auch seinen Abstand rom Auge zu kennen, weil schon die geringsten Aenderungen dieses Abstandes auf die Grösse des Netzhautbildes von merklichem Einfluss sind.

Bei hochgradiger Ametropie, bei welcher wenigstens für gewisse Entfernungen Correctionsgläser gebraucht werden müssen, tritt uns also das eigenthïmliche Verhalten entgegen, dass ein und dasselbe Auge nicht eine einzige bestimmte Sehschärfe besitzt, sondern verschiedene Sehschärfen, je nachdem wir es mit oder ohne Gläser untersuchen, und je nach der Art und der Brechkraft des verwendeten Glases und je nach seinem Abstand rom Auge.

Donders ${ }^{1}$ ) hat diejenige Sehschärfe, welche das emme-

1) Donders, Praktische Bemerkungen über den Finfluss von Hilfslinsen auf die Sehschärfe. Dieses Archiv XVIII. 2. S. 244 ff. (1872). 
Bemerkungen üb. d. Sehschärfe hochgradig myopischer Augen etc. 221

tropische Auge bei Accommodationsruhe und ohne Hilfe von Correctionsgläsern besitzt, als absolute Sehschärfe bezeichnet und die durch Accommodationsanspannung (deren Einfluss aber äusserst gering ist) oder durch Correctionsgläser erhaltene als relative Sehschärfe. Für das ametropische Auge nimmt er als absolute Sehschärfe ebenfalls die beim Fernsehen und bei Accommodationsruhe vorhandene an, wobei also Correctionsgläser zur Verwendung kommen; als relative Sehschärfe des myopischen Auges ist dann diejenige zu betrachten, welche beim Nahesehen ohne Gläser erreicht wird. Für die folgenden Betrachtungen dürte sich eine andere Bezeichnungsweise empfehlen; ich möchte diejenige Sehschärfe, welche irgend ein Ange, sei es emmetropisch oder ametropisch, ohne Hilfe von Correctionsgläsern besitzt, als wahre oder wirkliche Sehschärfe bezeichnen und die mit Hilfe von Correctionsgläsern erhaltene als corrigirte Sehschärfe. Handelt es sich z. B. um Myopie, wo wenigstens in gewissen Abständen auch ohne Glas deutlich gesehen werden kann, so ist es gewiss berechtigt, denjenigen Werth, wobei die Grösse des Netzhautbildes allein von der Brechkraft und Achsenlänge des Auges abhängt und nicht durch die verkleinernde Wirkung der Concavgläser reducirt ist, als wahre Sehschärfe zu bezeichnen. Bei Hypermetropie durch Aphakie wird man folgerichtig denjenigen Werth als den wahren betrachten müssen, bei welchem die vergrössernde Wirkung des Convexglases ansgeschlossen ist; das kann entweder dadurch erreicht werden, dass man den Abstand des Glases rom Auge möglichst gleich nuil macht, oder dass man seinen Einfluss durch Rechnung beseitigt.

Nach Abfassung dieser Zeilen finde ich bei Durchsicht der Literatur, dass schon Bordier'1), von einer gleichen

1) Bordier, Acuité visuelle des yeux amétropes. Acuité vraie et acuité apparente. Arch. d'Ophtalm. XIII. p. 355-371 (1894). 
Betrachtung ausgehend, den Ausdruck wirkliche Sehschärfe (acuité vraie) im gleichen Sinne wie oben gebraucht hat; für die mit Gläsern erhaltene beniützt er den Ausdruck scheinbare Sehschärfe.

Es ist klar, dass beim gewöhnlichen Gebrauch der Augen ebenso wohl die corrigirte als die wirkliche Sehschärfe zur Verwendung kommt und dass es von Interesse ist, beide zu kennen. Häufig begnügt man sich damit, die corrigirte Sehschärfe zu bestimmen; wo diese aber, wie bei hochgradiger Myopie, nicht mit der wirklichen zusammengeworfen werden darf, müsste dann die letztere durch Rechnung abgeleitet werden. Da aber die Entfernung der Gläser vom Auge schwer genau zu messen ist, hat Schweigger mit Recht empfohlen, bei hochgradiger Myopie die wirkliche Sehschärfe ohne Correctionsglas im Fernpunktsabstande direct zu bestimmen, oder, wo Correctionsgläser nicht zu vermeiden sind, dieselben dem Auge möglichst zu nähern, wobei ihr Einfluss auf die scheinbare Grösse des Gegenstandes am geringsten ist. Dass die directe Bestimmung der wirklichen Sehschärfe als allgemeine Methode überwiegende Nachtheile hat, ist von Donders in der oben citirten Arbeit dargethan worden; dies schliesst aber nicht aus, dass sie für gewisse Fälle, wie den hier vorliegenden, doch zu bevorzugen ist, wobei man sich eben mit ihren Mängeln abfinden muss. Es braucht hier kaum daran erinnert zu werden, dass zur Bestimmung der Sehschärfe bei einer so starken Annäherung, wie sie bei höchstgradiger Myopie erfordert wird, die meisten der gewöhnlich benutzten Sehproben nicht fein genug sind, und dass man sich daher der Burchardt'schen Punktproben oder äbnlicher bedienen muss, welche von dem Autor auch gerade dafür besonders empfohlen worden sind. Die feinste dieser Proben entspricht allerdings keinem kleineren Abstand als $15 \mathrm{~cm}$ rom Knotenpunkt, also einer Myopie von nicht ganz $7 \mathrm{D}$. Doch ist ja die Sehschärfe der meisten myopischen Augen erheblich 
Bemerkungen üb. d. Sehschärfe hochgradig myopischer Augen etc. 223

herabgesetzt, so dass man wohl nicht oft in die Lage kommen wird, sich nach noch feineren Probeobjecten umzusehen.

Wenn man, wie dies gewöhnlich geschieht, die Sehschärfe bei hochgradiger Myopie in grösserer Entfernung ermittelt mit Hilfe von Concavgläsern, die in einem gewissen Abstande vom Auge sich befinden, so erhält man reducirte Werthe derselben, während die später vorgenommene Prüfung des aphakischen Auges bei Emmetropie, wie weiter unten bewiesen werden soll, annähernd die wirkliche Sehschärfe ergiebt; bei Hypermetropie dagegen erhält man eine durch Convexgläser vergrösserte Sehschärfe. Die Unterschiede können, wie bekannt ist und sich unten noch genauer ergeben wird, ziemlich erhebliche sein. Es scheint mir aber nicht zweckmässig, wie auch Fukala neuerdings bemerkt hat, hier schlechthin von einer Verbesserung der Sehschärfe zu reden, weil die Sehschärfe bei demselben Auge je nach der Prüfungsmethode verschieden gross ausfällt und man somit lediglich in Folge der Verschiedenheit der angewandten Methode nach der Operation ein besseres Sehvermögen erhalten kann oder nicht. Man würde richtiger sagen, dass unter diesen oder jenen Umständen dem Auge eine grössere Quote oder der volle Betrag der wirklichen Sehschärfe oder ein Multiplum derselben zur Verfügung steht. Ist die Myopie durch die Operation zufällig gerade in Emmetropie verwandelt, so kann die Sehschärfe vor und nach der Operation ohne Correctionsgläser geprüft werden. Hängt in einem solchen Falle die Verbesserung des Sehens nur von Zunahme der Bildgrösse auf der Netzhaut $a b$, so kann bei der Prüfung ohne Gläser die Sehschärfe vor und nach der Operation gleich gefunden werden, bei Prüfung mit Gläsern aber vor der Operation kleiner, und nach der Operation grösser. Derartige Fälle sind natürlich besonders geeignet, um die Frage zu entscheiden, wie weit die nach der Myopie-Operation gefundene auffällige Besserung 
des Sehvermögens nicht nur auf Vergrösserung der Netzhautbilder, sondern auch auf Zunahme des Distinctionsvermögens des Auges bezogen werden muss.

Man muss sich also hüten, Verbesserung der Sehschärfe und Verbesserung des Distinctionsvermögens des Auges zusammenzuwerfen. Eine Verbesserung des Distinctionsvermögens bringt ceteris paribus immer eine Verbesserung der Sehschärfe mit sich; eine Zunahme der Sehschärfe beweist aber noch nicht ein grösseres Distinctionsvermögen. Die Sehschärfe kann besser werden, ohne dass das Distinctionsvermögen zunimmt, wenn z. B. das Auge durch Entfernung der Linse in den Stand gesetzt wird, beim Fernsehen anstatt der durch Concavgläser reducirten, seine wirkliche Sehschärfe zur Anwendung zu bringen oder dieselbe durch Vorsetzen von Convexgläsern noch zu steigern. Es muss aber bemerkt werden, dass, wenn hier auch die wirkliche Sehschärfe, wie sie oben definirt wurde, nicht erhöht und die Leistungsfähigkeit des Auges nicht gesteigert ist, das bezeichnete Verhalten rom praktischen Standpunkt aus doch als Verbesserung zu betrachten ist, da das Auge nach der Operation mehr leistet, da es Gegenstände unter einem kleineren Sehwinkel zu erkennen vermag als zuvor.

Der Betrag der auf Vergrösserung der Netzhautbilder beruhenden Zunahme der Leistungsfähigkeit des Auges lässt sich aus der Brennweite und dem Abstand des Correctionsglases unter Zugrundelegung der Werthe des reducirten Auges berechnen, wie aus der folgenden Darstellung zu entnehmen ist. Ist dieser Betrag bekannt, so ergiebt sich ans dem Vergleich mit den gemessenen Werthen der Sehschärfe, wie viel von der Zunahme der letzteren nicht auf Vergrösserung der Netzhautbilder bezogen werden kann, sondern einer Zunahme des Distinctionsvermögens zugeschrieben werden muss.

Obwohl das Problem des Einflusses von Brillengläsern auf die Grösse der Netzhautbilder schon mehrfach behandelt 
Bemerkungen üb. d. Sehschärfe hochgradig myopischer Augen etc. 225

worden ist, dürfte eine gerade für die vorliegende Frage berechnete Darstellung an dieser Stelle doch nicht überflüssig sein.

Eine allgemeine Formel, ans welcher der Finfluss der Correctionsgläser auf die Grösse der Netzhautbilder in allen vorkommenden Fällen zu berechnen ist, muss natürlich auch den Fall berücksichtigen, wo das Object sich in endlicher Entfernung befindet. Die Grösse des Netzhautbildes erscheint darin als Function der Tangente des Sehwinkels, die ja für alle Entfernungen angiebt, wie gross ohne Cor-

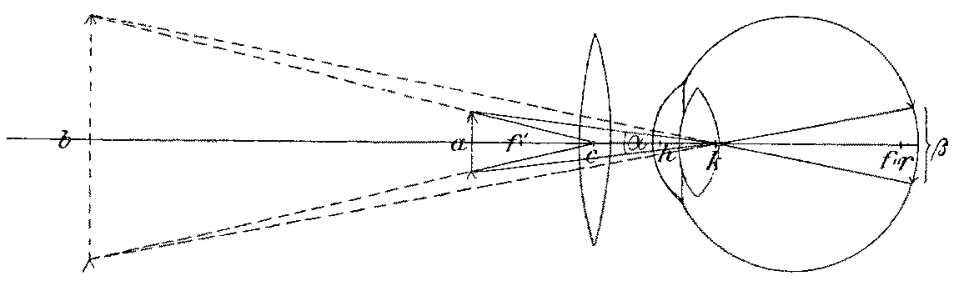

Fig. 1.

rectionsglas der Gegenstand dem Auge erscheint. Da es für die Rechnung bequemer ist, habe ich die Entfernungen nicht vom Hauptpunkt, sondern vom Knotenpunkt des Auges aus genommen, woran man sich vorkommenden Falles zu erinnern hat.

Es sei in Fig. 1:

$\beta$ die Bildgrösse auf der Netzhaut.

tga die Tangente des Sehwinkels des betrachteten Gegenstandes.

$f$ der Abstand $a c$ des Gegenstandes rom optischen Centrum des corrigirenden Glases.

$d$ der Abstand $c h$ des letzteren vom Knotenpunkt des Auges. $F$ die Brennweite des Glases (für convexe Gläser +, für concave - zu nehmen).

$F^{\prime}$ die vordere Brennweite $h f^{\prime}$

$F^{\prime \prime}$ die hintere Brennweite $h f^{\prime \prime}$ des Auges. Da es sich um ๆ. Graefe's Archiv firr Ophthalmologie. XIIII. 
Achsenmyopie handelt, können dafür die Werthe des reducirten Auges (15, resp. $20 \mathrm{Mm}$ ) benïtzt werden.

$\Phi^{\prime}$ die vordere Brennweite

$\Phi^{\prime \prime}$ die hintere Brennweite der Hornhaut, identisch mit der vorderen und hinteren Brennweite des aphakischen Auges. Alsdann gilt für corrigirende Convex- wie Concavgläser folgende Formel, welche aus den bekannten Brechungsformeln leicht abzuleiten ist:

$$
\beta=-\frac{F \cdot F^{\prime} \cdot(f+d)}{f F+(f-F) \cdot\left(F^{\prime \prime}-\mathrm{d}\right)} \cdot \operatorname{tg} \alpha
$$

Die von Fukala angegebene speciellere Gleichung lässt sich aus obiger herleiten, wenn man $f=\infty$ setzt und. berücksichtigt, dass darin der Abstand $d$ vom Hauptpunkt und nicht vom Knotenpunkt aus genommen ist.

Es seien nun der Reihe nach die verschiedenen vorkommenden Fälle betrachtet.

\section{Bildgrösse bei dem nicht-corrigirten myopischen Auge, Object im Fernpunktsabstand.}

In obiger Formel ist für diesen Fall zu setzen:

$$
F=\infty
$$

$d=0$

$f=\widetilde{F}$ (Abstand des Fernpunktes vom Knotenpunlst des Auges); dann erhält man

$$
\beta=-\frac{\mathfrak{F} \cdot F^{\prime}}{\widetilde{F}-F^{\prime \prime}} \cdot \operatorname{tg} \alpha
$$

Die Richtigkeit dieser Formel ergiebt sich unmittelbar aus der Brechungsformel $\frac{G^{\prime}}{g^{\prime}}+\frac{G^{\prime \prime}}{g^{\prime \prime}}=1$, da $\frac{\mathfrak{F} \cdot F^{\prime}}{\mathfrak{F}-F^{\prime \prime}}$ gleich der vom Knotenpunkt aus gemessenen hinteren Vereinigungsweite ist, wenn die vordere Vereinigungsweite $=\mathscr{F}$. Da die aus dem Fernpunkt kommenden Strahlen auf der Netzhaut vereinigt werden, ist also $\frac{\mathfrak{F} \cdot F^{\prime}}{\mathfrak{F}-F^{\prime \prime}}$ auch gleich der Entfernung der Retina vom Knotenpunkt des Auges. 
Da die Bildgrösse auf der Netzhaut der Entfernung der letzteren rom Knotenpunkt des Auges proportional ist, so nimmt sie natürlich bei verschiedenen Augen mit dem Grad der Myopie zu; sie wächst aber nicht in gleichem Maasse wie der Grad der Myopie, sondern langsamer, wie aus folgender kleiner Tabelle hervorgeht:

$$
\begin{aligned}
& \text { für } E \quad \text { ist } \beta=-15 \cdot \operatorname{tg} \alpha \\
& \text { " } M=1 D \text { " , " }-15,3, \\
& \text { " " ,5 " " , }-16,6 \text {, } \\
& \text { " , } 10, ", ",-18,7 \text {, } \\
& \text { " " , } 15, \text {, , , }-21,4 \text {, } \\
& \text { " ", } 20, ", ",-25 \quad \text {, } \\
& \text { " " } 25, ", "-30 \text { " }
\end{aligned}
$$

Bis zu ziemlich hohen Graden von Myopie ist also die von der Zunahme der Achsenlänge abhängige Vergrösserung des Netzhautbildes nur unbedeutend; sie beträgt z. B. bei $M=10 \mathrm{D}$ nur $1 / 4$ des Werthes für Emmetropie. Bei sehr hohen Graden steigt aber die Bildgrösse auf das Doppelte und mehr; man muss sich daher immer vergegenwärtigen, dass das hochgradig myopische Auge wegen seiner bedeutenden Achsenlänge, wenn es nur auf die Bildgrösse auf der Netzhaut ankäme, eigentlich eine erheblich bessere Sehschärfe zeigen müsste, als das emmetropische, und dass, wenn dies nicht der Fall ist, das Distinctionsvermögen herabgesetzt sein muss. In der That ist, wie Donders schon längst hervorgehoben hat, die Retina beim myopischen Auge wegen der Dehnung des Bulbus nicht die gleiche wie beim emmetropischen; eine gleich grosse Stelle wird voraussichtlich weniger percipirende Elemente enthalten, und wenn das Auseinanderrücken der letzteren im gleichen Maasse erfolgte, wie die Vergrösserung des Netzhautbildes, so könnten sich beide Einflüsse gerade compensiren. Da aber die Dehnung keine gleichmässige ist, die Netzhautelemente auch sonstige Schädigungen erfahren können, wir auch nicht wissen, ob im kindlichen Lebensalter bei dem Wachsthum des hinteren Bulbusabschnittes eine Vermehrung der percipirenden 
Elomente sicher auszuschliessen ist, lässt sich a priori über das Verhalten der Retina gar nichts sagen. Es würde aber von Interesse sein, zu untersuchen, ob sich durch genaue Prüfungen der Sehschärfe hierüber nicht gewisse Aufschlüsse erhalten liessen.

\section{Bildgrösse bei dem für $\infty$ Entfernung corrigirten myopischen Auge.}

In der allgemeinen Formel (I) ist für diesen Fall zu setzen

$$
\begin{gathered}
f=\infty \\
F=-F,
\end{gathered}
$$

man erhält dann

$$
\beta=-\frac{F \cdot F^{\prime}}{F+d-F^{\prime \prime}} \cdot \operatorname{tg} \alpha
$$

Die Myopie kann durch Gläser von verschiedener Brennweite corrigirt werden, wenn nur in jedem Falle der Brennpunkt der Concavlinse mit dem Fernpunkt des Auges zusammenfällt. Der Abstand der Linse vom Auge muss dabei immer gleich der Differenz zwischen dem Fernpunktsabstand und der Brennweite der linse sein.

oder

$$
\begin{aligned}
& d=\widetilde{\jmath}-F \\
& F=\mathfrak{F}-d .
\end{aligned}
$$

Führt man diesen Werth in die Gleichung III ein, so erhält man

$$
\beta=-\frac{(\mathscr{F}-d) F^{\prime}}{\widetilde{F}-F^{\prime \prime}} \cdot \operatorname{tg} c
$$

Da der Zähler des Bruches mit zunehmendem $d$ kleiner wird, so ergiebt sich aus der Formel: Wenn dieselbe Myopie durch Concavgläser ron verschiedener Brennweite, die sich in verschiedenem Abstand rom Auge befinden, corrigirt wird, so ist die Bildgrösse auf der Netzhaut um so kleiner, je weiter die Linse vom Auge absteht.

Könnte $d=0$ werden, $d . h$. das optische Centrum der 
Linse mit dem Knotenpunkt des Auges zusammenfallen, so wäre

$$
\beta=-\frac{\mathscr{J} \cdot F^{\prime}}{\mathfrak{F}-F^{\prime \prime}} \cdot \operatorname{tg} \alpha
$$

Die Bildgrösse wäre dann dieselbe wie bei dem nichtcorrigirten myopischen Auge. Will man also die wirkliche, d. h. die allein vom Auge abhängige Sehschärfe mit Hilfe von Gläsern bestimmen, so müssen diese, um die Fehler möglichst gering zu machen, so nahe es geht vor das Auge gehalten werden.

Ist $d=F^{\prime \prime}$, so wird

$$
\beta=-F^{\prime} \cdot \operatorname{tg} \alpha
$$

Diese Formel drückt den bekannten Satz aus, dass wenn das optische Centrum der Linse mit dem vorderen Brennpunkt des Auges zusammenfällt, die Bildgrösse auf der Netzhaut von der Brechkraft der Correctionslinse unabhängig ist und lediglich von der vorderen Brennweite des Auges abhängt. Der Werth $-F^{\prime} \cdot \operatorname{tg} \alpha$ entspricht zugleich der Bildgrösse bei dem emmetropischen Auge, wie sich aus IIIa ergiebt, wenn darin $\widetilde{F}=\infty$ und $d=0$ gesetzt wird.

Ist $d<F^{\prime \prime}$, so ist $\beta>F^{\prime} \cdot \operatorname{tg} \alpha$; ist $d>F^{\prime \prime}$, so ist $\beta<F^{\prime} \cdot \operatorname{tg} \alpha$, woraus sich wieder die Abnahme der Bildgrösse mit zunehmendem Abstand des Concavglases ergiebt.

Aus IIIc folgt, dass man zweckmässiger Weise die Correctionslinse in den vorderen Brennpunkt des Auges zu bringen hat, wenn verschiedene Augen mit ungleicher Achsenametropie auf ibre Sehschärfe vergleichend zu untersuchen sind. Die Prüfung der Sehschärfe hat hier den Zweck, ein Maass für das Distinctionsvermögen des Auges abzugeben; dies wird erreicht, wenn wir die Bildgrösse auf der Netzhaut in allen Fällen gleich machen, weil dann die Sehschärfe nur von dem Distinctionsvermögen abhängt. In der That wird bei der genannten Entfernung der Correctionslinse für denselben Sehwinkel die Bildgrösse auf der Netzhaut bei allen Augen die gleiche, einerlei welchen Refractions- 
zustand sie haben und welches Correctionsglas sie bedürfen, vorausgesetzt dass es sich um Achsenametropie handelt und dass die Werthe des reducirten Auges mit hinreichender Genauigkeit anwendbar sind.

Dies Verfahren ist aber nicht das richtige, wenn es sich darum handelt, das Distinctionsvermögen eines bestimmten Auges vor und nach einer Aenderung seiner Refraction zu vergleichen, denn die mit einem Concarglase erzielte Bildgrösse auf der Netzhaut ist unter allen Umständen kleiner und die mit einem Convexglase erzielte unter allen Umständen grösser als ohne Correction, so dass also dabei trotz gleichem Distinctionsvermögen die Sehschärfe verschieden ausfallen muss. Um gleichgrosse Netzhautbilder zu erhalten, muss man hier, wie oben schon angegeben wurde, die Augen ohne Gläser oder bei möglichst geringem, zu vernachlässigendem Abstande derselben vom Auge untersuchen. Man muss sich aber dabei daran erinnern, dass die Grösse der Netzhautbilder bei dieser Art der Bestimmung für verschiedene Augen von ungleichem Brechzustande verschieden ist, so dass die so gefundenen Werthe nur für ein und dasselbe Auge, nicht aber für verschiedene Augen vergleichbar sind.

Etwas Anderes ist es natïrlich, wenn man bei gleichem Distinctionsvermögen die Leistung eines Auges bei verschiedenen Arten der Correction durch Bestimmung der Sehschärfe prüfen will; dann giebt die Letztere ein Maass für die Grösse des Netzhautbildes ab.

Nimmt bei verschiedenen Augen der Grad der Myopie zu, während der Abstand der Correctionslinse rom Auge unverändert bleibt, so ergiebt sich das eigenthümliche Verhalten, dass die Bildgrösse auf der Netzhaut sich in entgegengesetzter Richtung ändert, je nachdem $d<$ oder $>F^{\prime \prime}$ ist.

Ist nämlich $d<F^{\prime \prime}$, so ist in der Formel IIIa der Bruch

$$
\frac{\mathfrak{F}-d}{\mathfrak{F}-F^{\prime \prime}}>1
$$

nimmt hierin $\widetilde{F}$ zu, also der Grad der Myopie ab, so nähert sich 
der Werth des Bruches der Einheit, nimmt also ab, somit bei wachsender Myopie zu.

Ist dagegen $d>F^{\prime \prime}$, so ist

$$
\frac{\mathfrak{F}-d}{\mathfrak{F}-F^{\prime \prime}}<1
$$

nimmt hierin $\widetilde{F} z u$, so nähert sich der Bruch ebenfalls der Einheit, sein Werth nimmt also zu, somit bei wachsender Myopie ab.

Für $d=F^{\prime \prime}$ ist, wie schon angeführt, die Bildgrösse auf der Netzhaut rom Grade der Myopie und der Correctionslinse unabhängig.

\section{Bildgrösse bei dem emmetropisch gewordenen aphakischen Auge.}

Die allgemeine Formel, in welcher für diesen Fall

$$
\begin{aligned}
f & =\infty \\
F^{\prime} & =\infty \\
d & =0 \\
F^{\prime} & =\Phi^{\prime}
\end{aligned}
$$

zu setzen sind, geht hier über in

$$
\beta=-\Phi^{\prime} \cdot \operatorname{tg} \alpha \text {. }
$$

Es fragt sich nun, welche Beziehung zwischen diesem Werthe und den Werthen für das myopische Auge anzunehmen ist.

$\Phi^{\prime}$, die vordere Brennweite des aphakischen Auges, ist $=$ der vorderen Brennweite der Hornhaut des myopischen Auges, da im ersteren nur noch die Brechung durch die Hornhaut übrig geblieben ist; ist das aphakische Auge emmetropisch, so befindet sich seine Retina in der hinteren Brennebene, $\Phi^{\prime}$ ist somit auch gleich dem Abstand der Retina vom Knotenpunkt des aphakischen Auges, welcher identisch ist mit dem Krümmungsmittelpunkt der Hornhaut. Nimmt man nun einen Krümmungsradius von $7 \mathrm{~mm}$ und einen Abstand des Hauptpunktes des myopischen Auges vom Hornhautscheitel von $2 \mathrm{~mm}$ an, so liegt der Knoten- 
punkt des myopischen Auges $2+5=7 \mathrm{~mm}$ hinter dem Hornhautscheitel und fällt mit dem Knotenpunkt des aphakischen Auges zusammen. Man kann daher ohne erheblichen Fehler annehmen, dass der Knotenpunkt seine Lage bei dem Verluste der Linse beibehält. Dann ist also $\Phi^{\prime}$ gleich dem Abstand zwischen Retina und Knotenpunkt des myopisehen Auges, der sich aus dem Fernpunktsabstand $\mathfrak{F}=g^{\prime}$ berechnen lässt:

$$
\begin{aligned}
\Phi^{\prime} & =g^{\prime \prime}=\frac{\mathfrak{F} \cdot F^{\prime}}{\mathfrak{F}-F^{\prime \prime},} \\
\text { also } & \beta=-\frac{\mathfrak{F} \cdot F^{\prime}}{\mathscr{F}-F^{\prime \prime}} \cdot \operatorname{tg} \alpha
\end{aligned}
$$

Dieser Werth ist nach II identisch mit dem des nichtcorrigirten, für seinen Fernpunkt eingestellten myopischen Auges; man hat daher den Satz:

Die Bildgrösse auf der Netzhaut bei dem durch Aphakie emmetropisch gewordenen Auge ist (nahehin) dieselbe, wie bei dem für seinen Fernpunkt eingestellten myopischen Auge vor der Operation.

Die vergleichende Untersuchung solcher Fälle kann also, wie schon oben bemerkt wurde, besonders empfohlen werden, um festzustellen, ob die Myopie-Operation nicht nur die Grösse der Netzhautbilder, sondern auch das Distinctionsvermögen des Auges beeinflusst, da sich gezeigt hat, dass die Sehschärfe, soweit sie lediglich von der Grösse der Netzhautbilder abhängt, in solchen Fällen vor und nach der Operation gleich sein muss.

Ist grössere Genauigkeit erwünseht, so kann man den Krtimmungsradius der Hornhaut des aphakischen Auges messen und daraus durch Multiplication mit 3 den Abstand der Retina von demselben erhalten und in Rechnung ziehen, da dieser gleich der vorderen Brennweite ist und $\Phi^{\prime}=\frac{n^{\prime}}{n^{\prime \prime}-n^{\prime}} \cdot x$, worin $n^{\prime}=1$, $n^{\prime \prime}=\frac{103}{77}$, somit $\frac{n^{\prime}}{n^{\prime \prime}-n^{\prime}}=2,96 \mathrm{zu}$ setzen sind. 
4. Bildgrösse bei dem hypermetropisch gewordenen aphakischen Auge mit Correction durch ein Convexglas für $\infty$ Entfernung.

Führt man in die allgemeine Formel die hier giltigen Werthe ein, nämlich:

$$
\begin{gathered}
f=\infty \\
F^{\prime}=\Phi^{\prime} \\
F^{\prime \prime}=\Phi^{\prime \prime}, \text { so erhält man } \\
\beta=-\frac{F \cdot \Phi^{\prime}}{F+\Phi^{\prime \prime}-d} \cdot \operatorname{tg} a
\end{gathered}
$$

Die Formel zeigt, dass hier umgekehrt wie bei Myopie $\beta$ um so grösser wird, je grösser $d$ ist, dass mit anderen Worten die Bildgrösse auf der Netzhaut mit dem Abstand der Convexlinse vom Auge zunimmt.

Könnte $d=0$ gemacht werden, so wäre

$$
\beta=-\frac{F \cdot \Phi^{\prime}}{\bar{F}+\Phi^{\prime \prime}} \cdot \operatorname{tg} \alpha
$$

Da in diesem Fall der (negative) Fernpunktsabstand $\mathfrak{F}$ des Auges gleich der Brennweite der corrigirenden Convexlinse ist

$$
\mathfrak{F}=-F
$$

so wird

$$
\beta=-\frac{\mathfrak{F} \cdot \Phi^{\prime}}{\widetilde{F}-\Phi^{\prime \prime}} \cdot \operatorname{tg} \alpha
$$

$\frac{\widetilde{F} \cdot \Phi^{\prime}}{\mathfrak{F}-\Phi^{\prime \prime}}$ ist aber die hintere Vereinigungsweite der aus dem (negativen) Fernpunkt kommenden Strahlen für die Brechung im aphakischen Auge, also, da das Bild auf die Retina fällt, auch gleich dem Abstand der Retina vom Knotenpunkt, welcher nach Formel II $\frac{\dot{\mathfrak{F}} \cdot F^{\prime}}{\widetilde{F}-F^{\prime \prime}}$ beträgt.

Könnte man also das corrigirende Convexglas in das Auge bineinsetzen, so würde die dabei erzielte Bildgrösse auf der Netzhaut der des emmetropisch-aphakischen und der des uncorrigirten myopischen Auges gleich sein und ihrem wirklichen Werthe entsprechen. 
Auch hier muss also zur Erlangung eines möglichst richtigen Resultates das Correctionsglas dem Auge thunlichst genähert werden, da mit zunehmendem Abstand sich die vergrössernde Wirkung des convexen Glases in immer höherem Maasse geltend macht und zu grosse Sehschärfen vortäuschen kann.

Ist der Abstand der Convexlinse vom Auge nicht gleich null, so ist für den Fernpunktsabstand zu setzen:

oder

$$
\mathfrak{F}=-(F-d)
$$

Die Formel $V$ wird daher:

$$
\beta=-\frac{(\mathfrak{F}-d) \Phi}{\widetilde{J}-\bar{\Phi}^{\prime \prime}} \cdot \operatorname{tg} \alpha
$$

Sie stimmt mit der für das myopische corrigirte Auge iiberein, wenn man in dieser für $F^{\prime}$ und $F^{\prime \prime} \Phi^{\prime}$ und $\Phi^{\prime \prime}$ setzt. Da aber $\widetilde{F}$ einen negativen Werth hat, so ist das Verıalten der Bildgrösse bei constantem Abstand der Linse, aber ungleichen Graden von Hypermetropie dem bei Myopie entgegengesetzt:

Wenn $d<\Phi^{\prime \prime}$ ist, so nimmt die Bildgrösse auf der Netzhaut mit zunehmender Hypermetropie ab;

ist aber $d>\Phi^{\prime \prime}$, so nimmt sie mit der Zunahme der Hypermetropie $z u$;

ist

so ist wieder

$$
\begin{gathered}
d=\Phi^{\prime \prime}, \\
\beta=-\Phi^{\prime} \cdot \operatorname{tg} \alpha,
\end{gathered}
$$

also von der Brechkraft der Linse unabhängig.

Wenn Schanz ${ }^{1}$ ) angiebt, die Grösse des Netzhautbildes nehme mit der Stärke des Staarglases $a b$, so ist dies zwar thatsächlich wohl in der Regel richtig, theoretisch aber nur, so lange der Abstand des Staarglases kleiner ist als die vordere Brennweite des Auges, was allerdings gewöhnlich der Fall ist.

\section{Bildgrösse bei dem trotz Aphakie myopisch gebliebenen Auge.}

Die Verhältnisse sind hier, wie sich leicht zeigen lässt, ganz dieselben wie bei dem mit Linse rersehenen myopischen Auge.

1) Loc. cit. S. 113. 
Bei Einstellung auf den Fernpunkt ohne Convexglas ist

$$
\beta=-\frac{\widetilde{\mho} \cdot \Phi^{\prime}}{\widetilde{F}-\Phi^{\prime \prime}} \cdot \operatorname{tg} \alpha
$$

worin $\widetilde{F}$ den Fernpunktsabstand und $\frac{\mathfrak{F} \cdot \Phi^{\prime}}{\mathfrak{F}-\Phi^{\prime \prime}}$ den Abstand der Retina rom Knotenpunkt bedeuten. Die Bildgrösse ist also auch hier (nach $V b$ ) der des unversehrt gebliebenen Auges (nahezu) gleich.

Bei Correction auf $\infty$ Entfernung durch ein Concarglas von der Brennweite $-F$ ist:

$$
\beta=-\frac{F \cdot \Phi^{\prime}}{F+d-\Phi^{\prime \prime}} \cdot \operatorname{tg} \alpha .
$$

Die Bildgrösse nimmt also auch in diesem Falle mit zunehmendem Abstand der Concavlinse vom Auge ab.

Die folgende Tabelle giebt eine übersichtliche Darstellung der Bildgrössen auf der Netzhaut bei gleichem Sehwinkel für eine Reihe von Fällen von Myopie, ohne Correction und mit Correction durch Concavgläser, bei verschiedenem Abstand derselben rom Auge, sowie für Emmetropie. Es ist dazu noch zu bemerken, dass die Werthe der ersten Reihe auch (nahehin) für das aphakische Auge gelten, wenn dasselbe durch die Entfernung der Linse gerade emmetropisch geworden ist. (Abgesehen von den Werthen der Bildgrösse ist daraus auch zu erkennen, wie sebr verschieden die Stärke der für die Correction höchstgradiger Myopie dienenden Concargläser bei ungleichem Abstand vom Auge sein kann.)

Die Tabelle zeigt, dass z. B. bei einer Myopie von $25 \mathrm{D}$, wenn das corrigirende Concavglas um die vordere Brennweite vom Hauptpunkt des Auges absteht $(d=20 \mathrm{~mm})$, die Bildgrösse auf der Netzhaut nur halb so gross ist, wie beim Sehen ohne Gläser. Hätte man also die Sehschärfe vor der Operation mit einem Concavglas in der angegebenen Entfernung vom Auge bestimmt, so würde man sie nach 


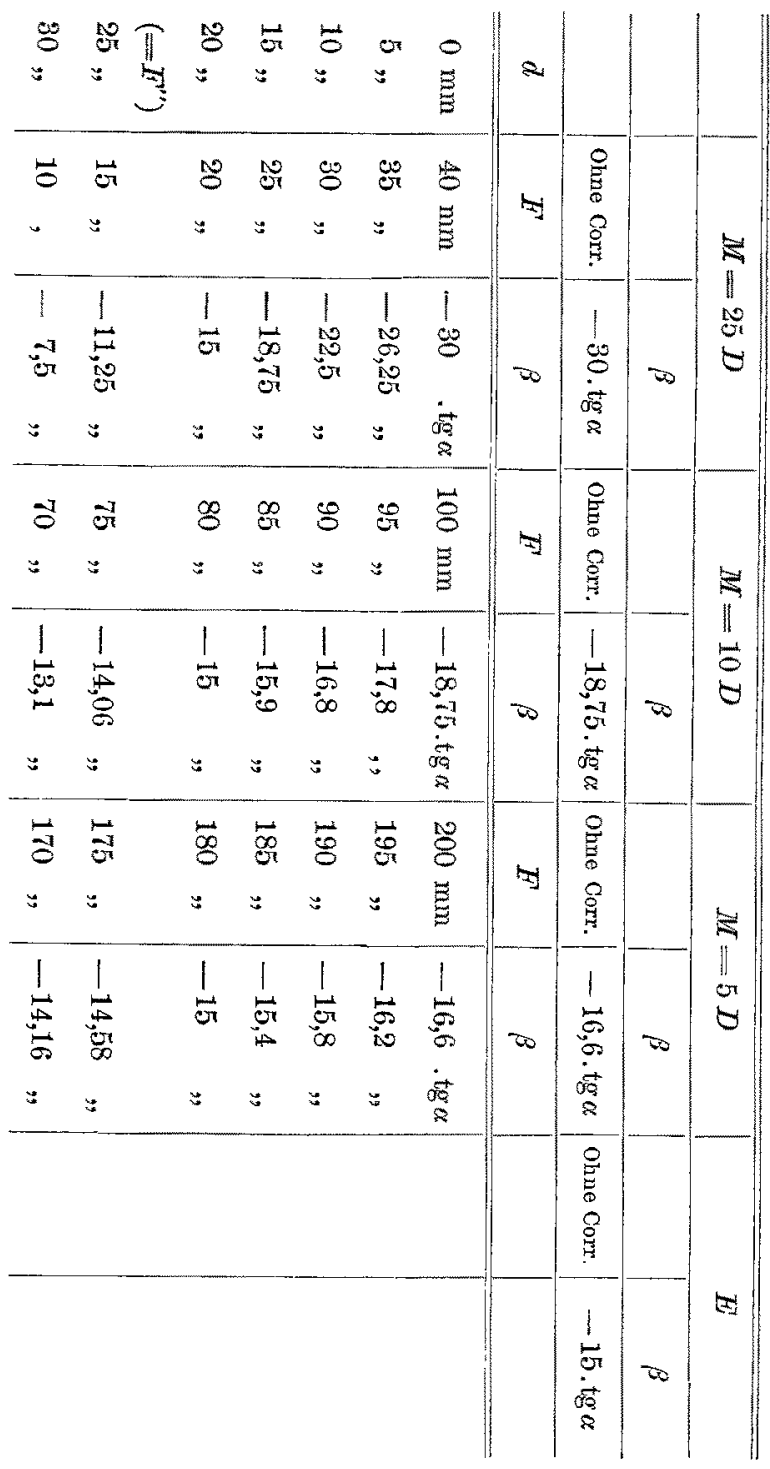

der Operation ceteris paribus doppelt so gross finden; sie würde aber der vor der Operation gefundenen gleich er- 
Bemerkungen üb. d. Sehschärfe hochgradig myopischer Augen etc. 237

scheinen, wenn man letztere ohne Concavglas im Fernpunktsabstande gemessen hätte.

Noch grössere Abstände der Concavgläser würden noch erheblichere Differenzen, also eine noch grössere Zunahme der für die Ferne verfügbaren Sehschärfe zur Folge haben.

Für eine Myopie von $30 \mathrm{D}$, die noch nicht den höchsten vorkommenden Grad darstellt, würde ferner die Bildgrösse bei directer Bestimmung 37,5 tg $\alpha$, bei indirecter, mit Correctionsglas in der vorderen Brennweite, nux $15 \operatorname{tg} \alpha$ betragen; der Unterschied kann also auf das $21 / 2$ fache und darüber steigen. Doch lassen sich Zunahmen der Sehschärfe um das 3 fache, entsprechend einer Myopie von 33,3 D; oder gar um das 4 fache, wofür eine $\mathrm{M}$ von $37 \mathrm{D}$ erforderlich wäre, wie Fukala schon anfangs richtig hervorgehoben hat, von etwaigen höchst seltenen Ausnahmen abgesehen, nur noch theilweise durch Vergrösserung der Netzhautbilder erklären. Von Fukala wird für die Mehrzahl der Fälle eine 2-4 fache, für einzelne eine 58 fache Zunahme und darüber berichtet; Besserungen bis zum 3 fachen beobachteten Sattler, Pflüger und Thier, bis zum 10 fachen v. Hippel. Da aber anzunehmen ist, dass in den bisher mitgetheilten Fällen die Sehschärfe in der Regel mit Concavgläsern und ohne Rücksicht auf deren Abstand rom Auge bestimmt wurde, so muss wenigstens ein erheblicher Theil der nach Extraction der Linse gefundenen Verbesserungen der Sehschärfe auf grössere Netzhautbilder bezogen werden.

Dass noch andere Umstände dabei mitwirken müssen, geht, abgesehen von den zu hohen Graden der erlangten Sehschärfe, auch aus dem Umstande hervor, dass, wie Fukala und andere Beobachter berichten, die Sehschärfe nach der Extraction noch längere Zeit allmählich zunimmt, so dass ihr Maximum erst nach mehreren Monaten oder erst nach Jahresfrist erreicht wird.

Fukala versucht diese nachträgliche Verbesserung der Sehschärfe auf Uebung des Auges zu beziehen, was mir aber nicht wahrscheinlich ist, da die Augen doch vorher 
stets in gewissem Gebrauch, also nicht ganz ausser Lebung waren, und da mir eine auf das Fernsehen beschränkte Uebung nicht annehmbar erscheint. Mit viel grösserem Rechte könnte man nach der Cataractoperation an einen Einfluss der Uebung denken, und doch sehen wir hier, dass nach beendigter Heilung alsbald ein den optischen Verhältnissen entsprechendes Sehvermögen erreicht wird. Die auch hier so häufig eintretende nachträgliche Besserung der Sehschärfe ist ja durch die Besserung der Transparenz- und Brechungsverhältnisse, insbesondere durch die Abnahme des operativen Hornbautastigmatismus vollkommen zu erklären. Die Analogie mit der Staaroperation legt vielmehr die Annahme nahe, dass auch bei operativer Correction der Myopie an der nachträglichen Besserung der Sehschärfe die gleiche Ursache betheiligt sein könne. So wenig dies innerhalb gewisser Grenzen zu bezweifeln ist, so lassen sich doch damit Besserungen, bei welchen die Sehschärfe grösser gefunden wird als bei Prüfung im Fernpunktsabstand vor der Operation, nicht oder nur ausnahmsweise erklären, wenn etwa ein vorherbestehender Hornhautastigmatismus durch die Operation guinstig beeinflusst wird.

7. Hippel bemerkt (loc. cit.), dass er sich die Thatsachen nur so deuten könne, dass durch die Aphakie all. mählich auch ein günstiger Einfluss auf die Netzhautfunction ausgeübt werde, lässt aber dahingestellt, auf welche Art sich derselbe vollzieht. Ich selbst kann Nichts zur Aufklärung dieser Frage beitragen, da in den in der hiesigen Klinik operirten Fällen, obwohl der Ausgang bei allen ein sehr befriedigender war, erhebliche Besserungen der Sehschärfe nur wenige Male beobachtet wurden, wo sie durch Vergrösserung der Netzhautbilder oder Besserung von Hornhautastigmatismus erklärt werden konnten.

Die für die Sehschärfe erlangten Resultate bei den zehn Augen, in denen die Behandlung zum Abschluss gekommen ist, stelle ich in der folgenden kleinen Tabelle zusammen: 
Bemerkungen üb. d. Sehschärfe hochgradig myopischer Augen etc. 239

\begin{tabular}{r|l|l|c|c}
\hline Nr. & \multicolumn{1}{|c|}{ Name } & S rorher & S nachher & Verhältniss \\
\hline 1 & Karl D. (L. A.) & $1 / 4$ & $1 / 3$ & $4 / 3$ \\
2 & Irna K. & $1 / 6$ & $1 / 3$ & 2 \\
3 & Marg. C. & $1 / 5-1 / 4$ & $1 / 4$ & $>5 / 4$ \\
4 & Marie R. & $1 / 5$ & $1 / 4$ & $5 / 4$ \\
5 & Ernst B. & $1 / 3$ & $1 / 3$ & 1 \\
6 & Fr. Kath. St. & $1 / 3$ & $1 / 7$ & $7 / 6$ \\
7 & Fr. C. & $1 / 8,8$ & $1 / 3$ & $3,3 / 3$ \\
8 & Elise M. & $1 / 10$ & $1 / 3$ & $10 / 3$ \\
9 & Anna N. & $1 / 3$ & $1 / 3$ & 1 \\
10 & Karl D. (R.A.) & $1 / 3$ & $1 / 3$ & 1 \\
& & & &
\end{tabular}

Es ist dazu zu bemerken, dass die Sehschärfe noch ohne Rücksicht auf die hier besprochenen Umstände vorzugsweise auf grösseren Abstand, wo nöthig mit Gläsern bestimmt wurde.

Im Fall 8 bestand vor der Operation ein Astigmatismus, dessen Correction durch Gläser gelungen war; der Schnitt wurde mit Rücksicht auf denselben nach unten angelegt und die Verbesserung der $S$ von $4 / 10$ auf $1 / 3$ ist daher mit Wahrseheinlichkeit - eine genane Messung konnte noch nicht stattfinden auf Verbesserung der Hornhantkrummung zu beziehen.

Es fragt sich nun noch, ob durch die Beseitigung der Linse auch Einflüsse erzeugt werden, welche unmittelbar eine nicht von Vergrösserung der Netzhautbilder, sondern von Zunahme des Distinctionsvermögens (im weitesten Sinn des Wortes) abhängige Verbesserung der Sehschärfe mit sich bringen.

Als ein möglicher Weise in dieser Richtung wirksames Moment ist früher von $S c h a n z^{1}$ ) und jetzt von Fukala eine Zunahme der Lichtstärke der Netzhautbilder in Betracht gezogen worden. Fukala kommt zu dem Ergebniss, dass das aphakische Auge gegenüber dem durch ein Concavglas corrigirten myopischen in dieser Hinsicht nicht im Vortheil, sondern im Gegentheil ein wenig im Nachtheil sei. In seinem kürzlich erschienenen Buche ${ }^{2}$ ) äussert er sich

1) Loc. cit.

2) Eukala, Heilung höchstgradiger Kurzsichtigkeit. Leipzig n. Wien. 8. 1896. S. 56 น. 57. 
aber ganz anders über den Einfluss der Gläser auf die Helligkeit der Netzhautbilder; er glaubt, dass diese bei dem durch Convexgläser corrigirten aphakischen Auge wesentlich heller seien als bei dem durch Concavgläser corrigirten myopischen und nimmt an, dass darauf ein grosser Theil der Verbesserung des Sehens nach der Myopie-Operation beruhe.

Schanz drückt sich sehr unbestimmt aus; obwohl er bemerkt, dass der grösseren Lichtmenge bei dem aphakischen Auge ein grösseres Netzhautbild entgegenwirke, so dass die Helligkeit "annähernd" dieselbe sei, giebt or am Schlusse seines Aufsatzes doch wieder an, dass die Lichtstärke zu Gunsten des aphakischen Auges beeinflusst werde.

Thatsächlich ist aber nach bekannten optischen Gesetzen die Helligkeit der Netzhautbilder unter den von den genannten Autoren gemachten Voraussetzungen beim Seben mit freiem Auge dieselbe wie beim Sehen durch ein corrigirendes Glas, es kann also nicht davon die Rede sein, Verschiedenheiten der Sehschärfe beim Sehen mit oder ohne Gläser durch geänderte Helligkeit der Netzhautbilder erklären zu wollen.

Die Richtigkeit der eben aufgestellten Behauptung ergiebt sich unter Anderen aus dem von Helmholtz ${ }^{1}$ ) bewiesenen Satz: „Wenn ein Beobachter durch ein centrirtes System brechender und spiegelnder Kugelflächen ein scharfes Bild eines leuchtenden Gegenstandes erblickt und wir den Verlust von Licht an den brechenden und spiegelnden Flächen vernachlässigen können, so erscheint jede Stelle des Bildes dem Beobachter ebenso hell, wie ihm die entsprechende Stelle des Gegenstandes ohne optische Instrumente gesehen erscheinen würde, so oft die ganze Pupille des Beobachters von den Strahlen getroffen wird, die von einem einzelnen Punkt jener Stelle ausgehen."

1) Physiol. Optik. 1. Aufl. S. 173 ff. 
Bemerkungen üb. d. Sehschärfe hochgradig myopischer Augen etc. 241

Der Beweis, den Helmholtz dafür liefert, ist ganz allgemein; er gilt für die Combination des Auges mit einem beliebigen System brechender Kugelflächen zu einem neuen System, muss also auch richtig sein für die Combination irgend eines Auges mit beliebigen Brillengläsern und nicht minder für die Combination des aphakischen Auges mit der Krystallinse. Es ist auch klar, dass die einzige dafür gestellte Bedingung stets zutreffen wird, dass nämlich immer die ganze Pupille ron den Strahlen getroffen wird, welche von einem einzelnen Punkte ausgehen, so lange man es mit den gewöhnlichen Dimensionen und Entfermungen der Correctionslinsen zu thun hat.

Dass die Helligkeit, mit welcher dem Auge ein Gegenstand erscheint, nicht geändert wird, wenn man ihn durch eine vorgehaltene Convexlinse betrachtet, kann man sich leicht auf folgende Art klar machen. Die Lichtmenge $\boldsymbol{M}$, welche von einem Flächenelemente des betrachteten Gegenstandes auf die Linse fällt, ist proportional der Grösse dieses Flächenelementes $a$ und der Helligkeit $H$ desselben und umgekehrt proportional seiner Entfernung von der Linse $f^{\prime}$; das virtuelle Bild des Flächenelementes, welches von der Linse entworfen wird, sendet aber eine Lichtmenge $\boldsymbol{M}^{\prime}$ aus, welche proportional ist seiner Grösse $b$ und umgekehrt proportional seiner Entfernung von der Linse $f^{\prime \prime}$; die Grösse des Bildes des Flächenelementes verhält sich aber zur Grösse des Flächenelementes selbst, wie die betreffenden Abstände von der Linse.

Also, wenn $H^{\prime}$ die Helligkeit des von der Linse entworfenen virtuellen Bildes bedeutet

$$
\begin{aligned}
& \frac{M}{M^{\prime}}=\frac{a \cdot H}{f^{\prime 2}}: \frac{b \cdot H^{\prime}}{f^{\prime \prime 2}} \text {. } \\
& \text { and } \quad \frac{a}{b}=\frac{f^{\prime 2}}{f^{\prime \prime 2}} \text {, } \\
& \text { also } \quad \frac{M}{M^{\prime}}=\frac{H}{H^{\prime}} \text {. }
\end{aligned}
$$

v. Graefe's Archiv für Ophthalmologie. XIIII. 
Nun muss aber $M=M^{\prime}$ sein, da nach der Voraussetzung die Absorption und Reflexion an der Linse zu vernachlässigen sind, somit ebensoviel licht auf die Linse fällt, als von ihr durchgelassen wird; somit ist auch

$$
H=H^{\prime}
$$

d. h. das durch die Convexlinse entworfene virtuelle Bild des Gegenstandes ist ebenso hell wie der Gegenstand selbst, wenn dieser ohne die Linse betrachtet wird.

Wie man sieht, kommt es bei dieser Betrachtung gar nicht auf die Weite der Pupille an, die erst bei der Betrachtung der Helligkeit der Netzhautbilder zu berücksichtigen ist; es würde hier nur die Apertur der Linse in Betracht zu ziehen sein; diese ist aber bei gewöhnlichen Glaslinsen immer so gross, dass durch sie die das Auge treffende Lichtmenge nicht verringert werden kann.

Dieselbe Betrachtung kann natürlich auch für die Brechung durch eine Concavlinse angestellt werden. Da somit die Helligkeit des durch eine Linse entworfenen Bildes der Helligkeit des Gegenstandes gleich ist, so ist es für das Auge dasselbe, ob man den Gegenstand durch eine Linse betrachtet oder ob man ihn in diejenige Entfernung bringt, in welche bei seiner ersten Lage das durch die Linse entworfene Bild fallen würde. Lässt sich nun noch zeigen, dass auch das Netzhautbild dieselbe Helligkeit besitzt, in welcher Entfernung vom Auge auch der Gegenstand sich befinden mag, so ist auch bewiesen, dass das Vorsetzen einer Linse die Helligkeit des Netzhautbildes überhaupt nicht beeinflusst.

Die Helmholtz'sche Beweisführung des soeben ererwähnten Satzes dürfte manchen Lesern einige Schwierigkeiten für das Verständniss bieten, es ist daher vielleicht erwünscht, im Folgenden einen etwas ausführlicheren Beweis desselben zu erhalten.

Die Lichtmenge $M$, welche von einem Flächenelemente des Gegenstandes auf die erste Hauptebene des Auges ge- 
langt, ist proportional der Helligkeit des Gegenstandes $I$; der Grösse des Flächenelementes $a$ und dem Querschnitt des von $a$ ausgehenden Strahlenbündels, wo dieses die erste Hauptebene trifft, $Q$ und umgekehrt proportional dem Quadrate der Entfernung des Gegenstandes von der ersten Hauptebene, $f^{\prime}$ (vgl. Fig. 2)

$$
M=\frac{H \cdot a \cdot Q}{f^{\prime 2}}
$$

Ist $b$ das Bild von $a$ auf der Netzhaut, so ist nach dem Gesetz der conjugirten Vereinigungspunkte auch $a$ das Bild von $b$; alles Licht, das von $a$ durch die Pupille in das Auge gelangt, vereinigt sich in $b$, es muss sich also auch alles Licht, das von $b$ ausgeht, in $a$ vereinigen.

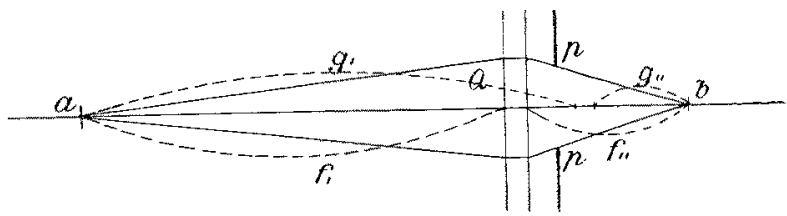

Tig. 2.

Dieselbe Lichtmenge $M$ wird nun auch die Retina erreichen. Die Helligkeit des Netzhautbildes $H^{\prime}$, welche dadurch erzeugt wird, können wir berechnen, da die Strahlen von jetat ab convergent verlaufen, indem wir uns vorstellen; dass dieselbe Tichtmenge $M$ ron der Netzhaut aus in entgegengesetzter Richtung durch die Pupille auf die zweite Hauptebene des Auges falle; wir erhalten dabei die Helligkeit des Netzhautbildes $H^{\prime}$, welche nöthig ist, um der zweiten Hauptebene die Lichtmenge $M$ zuzusenden; da diese aber der auf die Netzhaut fallenden Lichtmenge gleich ist, so ist $H^{\prime}$ auch gleich der Melligkeit des Netzhautbildes, wenn die Netzhaut von der Lichtmenge $\boldsymbol{M}$ getroffen wird.

Die Lichtmenge $M$ ist daher direct proportional der Helligkeit $H^{\prime}$ des Bildes, der Grösse desselben $b$, dem Querschnitt des Strahlenbündels in der zweiten Hauptebene $Q$, 
welcher dem in der ersten Hauptebene gleich ist, und umgekehrt proportional dem Quadrate der Entfernung $f^{\prime \prime}$ des Netzhautbildes $b$ von der zweiten Hauptebene

Somit ist

$$
M=\frac{H^{\prime} \cdot b \cdot Q}{f^{\prime \prime 2}}
$$

Nun ist aber auch

$$
H^{\prime}=\frac{M \cdot f^{\prime 2}}{b \cdot Q} \text {. }
$$

$$
M=\frac{H \cdot a \cdot Q}{f^{\prime 2}}
$$

daher

$$
H^{\prime}=\frac{H \cdot a \cdot Q \cdot f^{\prime \prime 2}}{b \cdot Q \cdot f^{\prime 2} \cdot}=\frac{H \cdot a \cdot f^{\prime \prime 2}}{b \cdot f^{\prime 2}} \text {. }
$$

Der Werth von $\frac{a \cdot f^{\prime \prime 2}}{b \cdot f^{\prime 2}}$ ist nun folgendermaassen zu. berechnen:

$$
\text { Bekanntlich ist } \quad \frac{a}{b}=\frac{g^{2}}{g^{\prime 2}},
$$

worin $g^{\prime}$ und $g^{\prime \prime}$ die Entfernungen des Gegenstandes und seines Bildes rom vorderen, resp. hinteren Knotenpunkt des Auges bedeuten; (die Werthe von $g^{\prime}$ und $g^{\prime \prime}$ sind auf das Quadrat zu erheben, da $a$ und $b$ Flächenelemente sind).

Nun ist nach bekannten optischen Gesetzen:

$$
\begin{aligned}
& \frac{g^{\prime}}{g^{\prime \prime}}=\frac{-g^{\prime \prime} \cdot G^{\prime}}{g^{\prime \prime}-G^{\prime \prime}}: g^{\prime \prime}=\frac{-G^{\prime}}{g^{\prime \prime}-G^{\prime \prime}}, \\
& \frac{g^{\prime}}{g^{\prime \prime}}=\frac{-G^{\prime}}{f^{\prime \prime}-\left(F^{\prime \prime}-F^{\prime}\right)-G^{\prime \prime}}=\frac{-F^{\prime \prime}}{f^{\prime \prime}-F^{\prime \prime}+F^{\prime}-F^{\prime}}=\frac{-F^{\prime \prime}}{f^{\prime \prime}-F^{\prime \prime}}, \\
& \text { somit } \\
& \qquad \frac{a}{b}=\frac{F^{\prime \prime 2}}{\left(f^{\prime \prime}-\overline{F^{\prime \prime}}\right)^{2}} .
\end{aligned}
$$

Fiihrt man diesen Werth von $\frac{a}{b}$ in die obige Formel ein, so erhält man:

$$
\begin{gathered}
H^{\prime}=H \cdot \frac{F^{\prime \prime 2} \cdot f^{\prime 2}}{\left(f^{\prime \prime}-F^{\prime \prime 2}\right) \cdot f^{\prime 2}} . \\
\text { Nun ist } \quad f^{\prime}=\frac{f^{\prime \prime} F^{\prime}}{f^{\prime \prime}-F^{\prime \prime}},
\end{gathered}
$$


Bemerkungen üb. d. Sehschärfe hochgradig myopiseher Augen etc. 245

somit

$$
H^{\prime}=\frac{H \cdot F^{\prime \prime 2} \cdot f^{\prime \prime 2}}{f^{\prime \prime 2} \cdot F^{\prime 2}}=H \cdot \frac{F^{\prime \prime 2}}{F^{\prime 2}}
$$

Nun ist weiter

$$
\begin{aligned}
& \frac{F^{\prime 2}}{F^{\prime 2}}=\frac{n^{\prime 2}}{n^{\prime 2}}, \\
& H^{\prime}=H \frac{n^{\prime 2}}{n^{\prime 2}} .
\end{aligned}
$$

also

Die Formel besagt, dass wenn der betrachtete Gegenstand die Helligkeit $H$ besitzt, seinem Netzhautbild die Helligkeit $H^{\prime}=H \frac{n^{\prime \prime 2}}{n^{\prime 2}}$ zukommt, und umgekehrt, dass also bei einer gegebenen Weite der Pupille die Helligkeit des Netzhautbildes lediglich von der Helligkeit des Gegenstandes und von dem Brechungsverhältniss des ersten und letzten Mittels abhängt, nicht aber von der Entfernung des Gegenstandes, so lange die anfangs gemachte Voraussetzung zutrifft, dass der Querschnitt des von einem Flächenelement des Gegenstandes ausgehenden Strahlenbündels nur durch die Pupille beschränkt wird, so dass also die ganze Pupille von demselben ausgefüllt ist.

Wie man sieht, kommt bei dieser Betrachtung die Weite der Pupille zur Geltung in der Grösse $Q$, welche den Querschnitt des von der Pupille durchgelassenen Strahlenbündels bedeutet, wo dieses die Hauptebenen des Auges schneidet. Da $Q$ sowohl im Nenner als im Zähler vorkommt, so fällt es heraus und das Resultat wird ron der als constant angenommenen Weite der Pupille unabhängig.

Wenn man dagegen die Helligkeit der Netzhautbilder unter sonst gleichen Verhältnissen bei verschiedener Pupillenweite vergleicht, so erweist sie sich natürlich von der letzteren abhängig und zwar ist sie dem Quadrat des Pupillendurchmessers einfach proportional.

Die scheinbar so einlenchtende Darstellung von Schanz, welche Fukala in obigem Aufsatz weiter ausgeführt hat, muss sonach einen Fehler enthalten, der wohl in Folgendem zu suchen ist.

Schanz betrachtet den Einfluss, den die Brechung in einer Glaslinse auf die Helligkeit der Netzhautbilder ausübt, indem er 
die Querschnitte, welche die durch die Pupille gehenden Strahlenbündel mit der Ebene der Linse bilden, bei freier Betrachtung und beim Sehen durch die Linse vergleicht. Für die Brechung in der Linse an sich kommt aber die Weite der Pupille nicht in Betracht; es ist vielmehr zunächst die Helligkeit des durch die Linse entworfenen Bildes mit der des Gegenstandes zu vergleichen. Beide sind, wie sich gezeigt hat, gleich, weil die dem Bilde zukommende Lichtmenge in dem gleichen Verhältrisss kleiner oder grösser ist als die Bildgrösse. Dasselbe gilt auch wieder für die Brechung im Auge: das näher gelegene Bild sendet der Netzhaut mehr Licht; im gleichen Verhältniss wird aber auch das Netzhautbild grösser, so dass auch hier ein völliger Ausgleich stattindet.

Es ist daher anch ohne Belang, wenn Schanz, wie than Fukala anseheinend mit Recht vorwirft, bei seiner Rechnung aus Versehen nicht die quadratischen, sondern die linearen Dimensionen der Netzhautbilder zur Division benutzt haben sollte, was bei der Kürze der Darstellung von Schanz schwer ganz sicher festzustellen ist.

Schanz hat als weiteren Umstand, welcher günstig auf die Sehschärfe des aphakischen Auges einwirken kömne, angeführt, dass die Lichtzerstreuung bei demselben geringer sei, weil die Pupille bei gleicher Grösse mehr von den Randstrahlen abschneide, wenn die Strablen, wie bei Aphakie, stärker convergirend auf sie fallen, als wenn sie durch ein vorgesetztes Convexglas bei Myopie eine geringere Convergenz erhalten haben.

Eine ähnliche Betrachtung, wie für die Lichtstärke zeigt zunächst, dass wenn das Auge dasselbe ist, die Correctionsgläser die Schärfe des auf der Netzhaut entworfenen Bildes nicht beeinflussen.

Es sei in Fig. $3 a$ ein in der Axe gelegener Punkt des leuchtenden Gegenstandes und $b$ sein Bild auf der Netzhaut. Die obere Hälfte der Zeichnung versinnlicht den Gang der Strahlen bei directer Betrachtung des Gegenstandes, die untere Hälfte für den Fall, wo ein in unendlicher Entfernung befindlicher Gegenstand $a^{\prime}$ durch eine Concavlinse betrachtet wird, welche von ihm ein virtuelles 
Bemerkungen üb. d. Sehschärfe hochgradig myopischer Augen etc. 247

Bild in $a$ entwirft. Der von $a$ ausgehende Lichtstrahl, welcher nach dem von der Hornhaut entworfenen Bilde $p^{\prime}$ des Pupillenrandes $p$ hin gerichtet ist, wird nach einem bekannten optischen Gesetz durch die Hornhaut so gebrochen, dass er eben durch die Pupille hindurchgeht. Derselbe Strahl wird alsdann durch die Linse so gebrochen, als ob er, rückwärts verlängert von dem durch die Linse entworfenen Bilde $p^{\prime \prime}$ des Pupillenrandes herkäme. Dieses Bild liegt (wie Helmholtz berechnet hat ${ }^{1}$ ); ca. 0,113 mm hinter der Pupillarebene (retinawärts davon) und ist um ca. ${ }^{1 / 18}$ vergrössert.

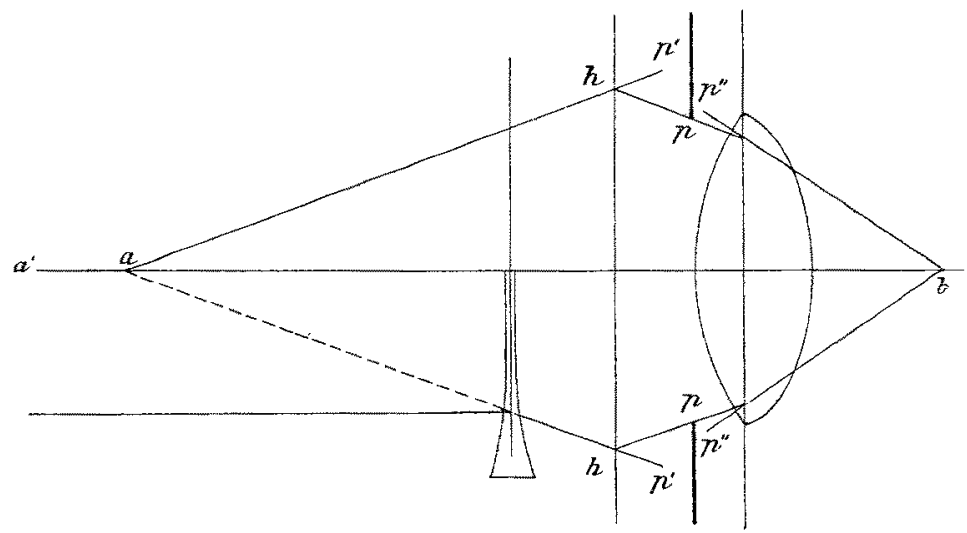

Fig. 3 .

Die Schärfe der Netzhautbilder hängt (bei möglichst genauer Accommodation) von dem Grade des vorhandenen Astigmatismus (im weitesten Sinn des Wortes) und von der Weite der Pupille ab. In Bezug auf die Lichtbrechung sind die Verhältnisse in beiden durch Fig. 3 dargestellten

1) Helmholtz, Physiol. Optik, 1. Auf. S. 98. (Es darf nicht anffallen, dass das Bild, obwohl es ein virtuelles ist, im Verhäitniss zu der vorderen Fläche der Linse nach hinten verschoben ist; es liegt zwar vor dem zweiten Hauptpunkt, aber hinter der vorderen Fläche der Linse, weil diese noch etwas weiter nach vorn von dem zweiten Hauptpunkt entfernt ist.) 
Fällen genau dieselben, da die Strahlen beide Male in Bezug auf das Auge von demselben Punkte ausgehen und sich in demselben Punkte schneiden. Aber auch für die Pupille findet kein Unterschied statt. Erhält die Netzhaut kein punktförmiges Bild, indem sich ein Theil der Strahlen vor oder hinter ihr schneidet, so ist für den Durchmesser des Zerstreuungskreises die Grösse des Linsenbildes der Pupille maassgebend, von welchem ja die Strahlen herzukommen scheinen. Da nun dieses Bild, wie auch sein Abstand von der Netzhaut, in beiden Fällen gleich ist, so ist auch die Diaphragma-Wirkung der Pupille dieselbe.

Dagegen findet in dem von Schanz betrachteten Falle, wo das optische System des Auges beide Male nicht dasselbe ist, allerdings ein kleiner Unterschied statt, z. B. bei dem Vergleich zwischen dem aphakischen und dem durch ein Concavglas corrigirten myopischen Auge, doch sind die Verhältnisse etwas complicirt, wie aus Fig. 4 zu ersehen ist. In Bezug auf die Wirkung der Pupille ist, wie Schanz richtig betont hat, das aphakische Auge ein wenig im Vortheil gegenüber dem corrigirten myopischen Auge. Für das erstere kommt, da die Linse fehlt, die Pupille $p p$ selbst als Diaphragma in Betracht, für das Letztere ihr von der Linse entworfenes Bild $p^{\prime \prime} p$; die Verschiebung desselben nach hinten und die Vergrösserung wirken in gleichem Sinn, um die Zerstreuungskreise auf der Netzhaut etwas zu vergrössern. Der Unterschied ist aber so gering, dass er gegeniiber den viel erheblicheren anderen Umständen, welche die Deutlichkeit des Bildes beeinflussen können, wohl kaum in Betracht kommt; sieht man doch auch sonst nicht, dass kleine Unterschiede in der Weite der Pupille, sobald das Auge für die Entfernung des Gegenstandes accommodirt ist, was ja hier vorausgesetzt wird, eine merkliche Aenderung der Sehschärfe bedingen.

In Bezug auf die Strahlenbrechung ist das Verhalten in beiden Fällen das Folgende: 
Bemerkungen üb. d. Sehschärfe hochgradig myopischer Augen etc. 249

Wie die obere Zeichnung versinnlicht, ist bei dem aphakischen Auge der Querschnitt des von a ausgehenden und in $b$-zur Vereinigung kommenden Strahlenbündels mit der Hornhaut $=h h$. Bei dem myopischen Auge (untere Zeichnung) entwirft zunächst die Concavlinse von $a$ das virtuelle Bild $a^{\prime}$, von diesem die Hornhaut das reelle Bild $b^{\prime}$ und endlich von dem letzteren die Krystallinse das an derselben Stelle, wie bei dem aphakischen Auge liegende Bild $b$. Der Querschnitt des von $a$ ausgehenden und nach $p^{\prime} p^{\prime}$, dem Hornhautbilde der Pupille gerichteten Strahlenbündels

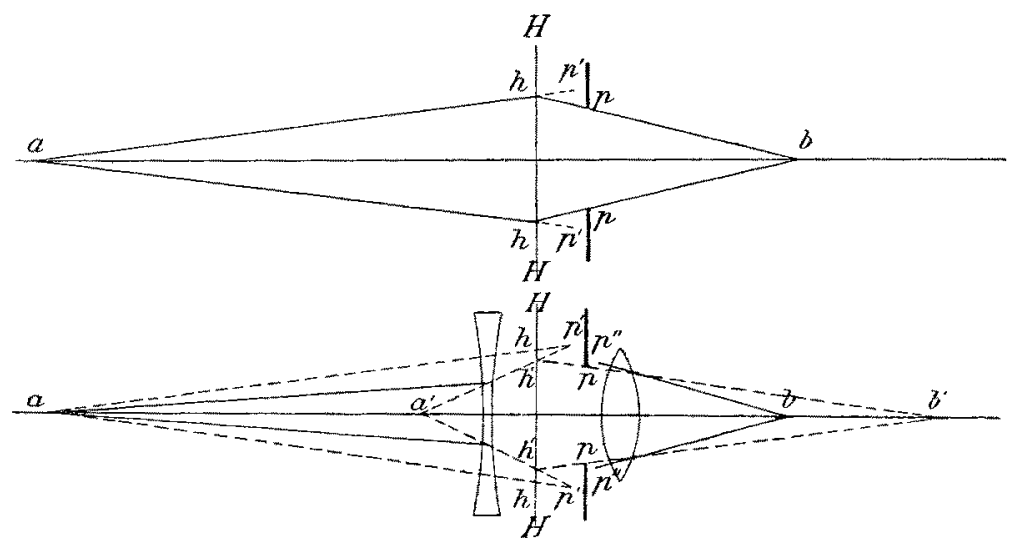

Fig. 4.

mit der vorderen Hornhautfläche ist hier $h^{\prime} h^{\prime}$; man sieht unmittelbar, dass $h^{\prime} h^{\prime}$ kleiner ist als $h h$. Das in das aphakische Auge eindringende Strahlenbiundel enthält also Strahlen, welche die erste Trennungsfläche des Auges weiter entfernt von der Achse treffen, als bei dem myopischen; dies kann nun ebenfalls von einem gewissen geringen Einfluss sein, der sich freilich schwer berechnen lässt, aber miudestens zuweilen zu Ungunsten des aphakischen Auges ausfallen kann.

Auch die sonst noch von Schanz zu Gunsten des aphakischen Auges angeführten Umstände, der Wegfall 
eines Mangels an Centrirung der Krystalllinse und der Reflexe an den Oberflächen der letzteren dürften nicht als erheblich anzuschlagen sein, da doch die Sehschärfe auch bei ideal gelungener Beseitigung einer traumatischen Cataract bei einem jugendlichen Individuum nicht leicht grösser gefunden wird als 1. Im Gegensatz hierzu werden ja selbst durch Operationen, die wir als vollkommen gelungene betrachten, recht oft Umstände herbeigeführt, welche die Deutlichkeit der Netzhautbilder in dieser oder jener Richtung viel erheblicher beeinflussen, wie z. B. Kapseltrübungen und Aenderungen der Hornhautkrümmung, so dass sich über den Einfluss der Myopie-Operation auf die Schärfe der Netzhautbilder etwas Allgemeines wohl nicht aussagen lässt.

Ich komme also zu dem Ergebniss, dass eine irgend erhebliche Verbesserung der Sehschärfe, nach operativer Beseitigung einer nicht mit Trübung der Linse complicirten Myopie, welche nicht auf' Vergrösserung der Netzhautbilder durch die Wirkung der Correctionsgläser beruht, wohl kaum auf Verbesserung der optischen Verhältnisse bezogen werden kann, und ich sehe sonach keine Möglichkeit, sie anders als durch einen Einfluss auf die Retina zu erklären. Es wäre natürlich ron grosser Wichtigkeit, wenn sich ein solcher mit Sicherheit feststellen liesse und ich hoffe durch obige Bemerkungen dazu anzuregen, dass darüber weitere, möglichst genaue Beobachtungen angestellt werden.

Zum Schluss möchte ich noch kurz auf den alten, zuerst von Vierordt aufgestellten Vorschlag eingehen, für welchen in newerer Zeit besonders Guillery ${ }^{\mathbf{1}}$ ) wieder eingetreten ist, und der von Fukala bei dieser Gelegenheit ebenfalls zur Sprache gebracht wird, ob es nicht richtiger wäre, anstatt der linearen Grösse der Netzhautbilder deren Flächenausdehnung als Maass der Sehschärfe zu benützen.

1) Guillery, Ein Vorschlag zur Vereinfachung der Sehproben. Archiv für Augenheilkunde. XXIII. (1891); Derselbe, Ueber die räumlichen Beziehungen des Licht- und Farbensinnes. Ibid. XXXI. (1895.) 
Für die Frage, wie die Verbesserung der Sehschärfo nach der Myopie-Operation zu erklären ist, hat natürlich die Entscheidung dieser Controverse keine Bedeutung, da ja die concreten Verhältnisse dieselben bleiben, mit welchem Maasse man sie auch messen mag.

Was die Sache selbst angeht, so glaube ich, dass der Vierordt'sche Vorschlag sich in Zukunft so wenig als bisher Fingang in die Praxis verschaffen wird, da die Numerirung der Sehproben nach Flächenmaass die Bestimmung der Sehschärfe nicht erleichtern kann, sondern eher unbequemer machen würde. Mir scheint der Vorschlag aber auch nicht theoretisch richtig zu sein. Die Bestimmung der Sehschärfe geht in ihrer einfachsten Form auf die Messung des kleinsten Abstandes zuriick, bei welchem zwei Punkte noch getrennt gesehen werden, sie benützt also áls Maass für die Leistung des Auges lineare Dimensionen und nicht Flächen. Es ist zwar richtig, dass das Auge, wie Fukala bemerkt, keine Punkte oder Linien, sondern streng genommen Flächen sieht. Auch was wir beim Sehen als Punkte bezeichnen, sind Raumempindungen, denen der Charakter der Ausdehnung untrennbar anhaftet, wobei nur diese Ausdehnung so gering ist, dass sie nicht weiter verkleinert werden kann, weil sie an der Grenze der Leistungsfähigkeit 'des Auges liegt. Was wir aber messen, ist nicht die Ausdehnung dieser Elemente der räumlichen Empinindung, sondern der Abstand zweier materieller Punkte, die eben noch zwei getrennte Empfindungen hervorrufen. Diese materiellen Punkte können wir uns ohne Fehler als Punkte im mathematischen Sinn des Wortes vorstellen, weil Objecte, deren räumliche Ausdehnung verschwindend klein ist, wie die Sterne, bei hinreichender Helligkeit eine Empfindung hervorrufen können, die aber als solche nicht punktförmig ist, sondern wie jede Gesichtsempfindung den Charakter des Flächenhaften an sich trägt. 